\title{
Recurrence of equine coital exanthema in thoroughbred stallions
}

\author{
Jaehyuk Yang ${ }^{1}$, Yoon-Kyu Lim $^{2, *}$ \\ ${ }^{1}$ Department of Equine industry, Korea National College of Agriculture and Fisheries, Hwaseong 445-760 Korea \\ ${ }^{2}$ College of Veterinary Medicine and Veterinary Medical Research Institute, Jeju National University, Jeju 690-756, Korea \\ (Received: December 28, 2011; Revised: April 28, 2013; Accepted: July 1, 2013)
}

\begin{abstract}
The object of this study was to evaluate recurrence of equine coital exanthema (ECE) whether re-infection or re-activation of causative virus. ECE is a venereal disease of horses caused by equine herpesvirus type 3 (EHV3). Like other herpesviruses, it may persist in infected horses for a long time. There is a controversy on the cause of ECE as the recurrence or the reinfection. This disease had occurred firstly on stallions and broodmares in Korea. The horses had rebreeded after healing routinely. Next year, the disease recurrented on the just same affected horses among stallions. The result of this study, re-outbreak of ECE in stallions is recurrence of ECE, but not reinfection of the virus.
\end{abstract}

Keywords : equine coital exanthema, equine herpesvirus type 3, recurrence

Equine coital exanthema (ECE) is a venereal disease of horses caused by equine herpesvirus type 3 (EHV-3). Lesions generally are confined to the external genitalia of stallions and mares. The penis and prepuce are the most commonly affected areas in stallions. Lesions begin as small, discrete, circumscribed vesicles or pustules that often become confluent [2]. Spread of EHV-3 infection without coitus has been described. Typically, an inapparently infected mare transmits the virus to a stallion at the time of breeding. Diagnosis usually can be made on the basis of the characteristic clinical signs in mares and stallions. Healing occurs in a few weeks, often leaving depigmented spots. Recurrent coital exanthema usually occurs in aged broodmares but may also occur in stallions. The relationship between viral recrudescence and recurrent coital exanthema in the equine is unknown [1]. Reoccurrences of ECE are seen, especially after periods of stress [6]. Some equine herpesviruses are recurrent due to reinfection and reactivation [3]. Like other herpesviruses, it may persist in infected horses for a long time. Immune carriers may have no clinical signs [4]. The disease may reappear the next season during times of stress or may not be apparent for several years, if ever again [5]. However, nothing is known about the persistence of EHV-3, but it is assumed [7]. There is a controversy on the cause of ECE as the recurrence or the reinfection.

ECE had occurred firstly on Thoroughbred stallions and broodmares in Republic of Korea in 2005. Two stallions of Korea Racing Authority and many broodmares had expressed typically clinical signs. In addition, the stallions were confirmed by the laboratorial examination $[8,9]$. The stallions had rebreeded routinely after healing routinely and had not clinical signs during breeding season. In 2006, ECE had recurrent same affected two stallions. The stallions showed clinical signs such as vesicles and pustules on the penis at the

Table 1. Outbreak of ECE in stallions belong to Korea Racing Authority

\begin{tabular}{ccccc}
\hline \hline \multirow{2}{*}{ Symbol of stallions } & \multicolumn{2}{c}{$2005^{*}$} & & 2006 \\
\cline { 2 - 5 } & Clinical signs & PCR & Clinical signs & PCR \\
\hline KS I & Positive & Positive & Positive & Positive \\
KS II & Positive & Positive & Positive & Positive \\
KS III & Negative & Negative & Negative & Not done \\
KS IV & Negative & Negative & Negative & Not done \\
The rest of the stallions & Negatives & Not done & Negatives & Not done \\
\hline
\end{tabular}

*Yang and Lim [9]. PCR: polymerase chain reaction.

*Corresponding author

Tel: +82-64-754-3367, Fax: +82-64-756-3354

E-mail: yklim@jejunu.ac.kr 
time of breeding. The diagnosis of ECE was carried out by polymerase chain reaction in the same methods in 2005 cases (Table 1).

In conclusion, we are considered that this re-outbreak of ECE in stallions is recurrence of ECE, but not reinfection of the virus.

\section{Acknowledgments}

This research was supported by the 2013 scientific promotion program funded by Jeju National University.

\section{References}

1. Blanchard TL, Kenney RM, Timoney PJ. Venereal disease. Vet Clin North Am Equine pract 1992, 8, 191-203.

2. Colahan PT, Merritt AM, Moore JN, Mayhew IG.
Manual of equine medicine and surgery. 1st ed. pp. 307309, Mosby, Missouri, 1999.

3. Field HJ, Awan AR, de la Fuente R. Reinfection and reactivation of equine herpesvirus-1 in the mouse. Arch Virol 1992, 123, 409-419.

4. Neely DP. Current therapy in theriogenology. 1st ed. pp. 694-706, WB Saunders, Philadelphia, 1980.

5. Scott DW. Large animal dermatology. 1st ed. pp. 1-4, WB Saunders, Philadelphia, 1988.

6. Scott DW, Miller WH Jr. Equine dermatology. 1st ed. pp. 382-383, Saunders, St. Louis, 2003.

7. Studdert MJ. Virus infections of equines. 1st ed, pp. 3946. Elsevier, Amsterdam, 1996.

8. Yang J, Lim YK. Characteristics on equine herpesvirus type 3 from Korea. J Life Science 2011, 21, 1156-1162.

9. Yang J, Lee K, Lim YK. Evaluation of clinical signs on equine coital exanthema in Thoroughbred horses. J Vet Clin 2011, 28, 369-374. 